\title{
Spreading Dynamic of a PLSGP Giving up Smoking Model on Scale-Free Network
}

\author{
Yanling Fei*, Xiongding Liu \\ School of Electronics and Information, Yangtze University, Jingzhou, China \\ Email: ^201771338@yangtzeu.com.cn
}

How to cite this paper: Fei, Y.L. and Liu, X.D. (2018) Spreading Dynamic of a PLSGP Giving up Smoking Model on Scale-Free Network. Open Access Library Journal, 5: e4365.

https://doi.org/10.4236/oalib.1104365

Received: January 23, 2018

Accepted: February 20, 2018

Published: February 23, 2018

Copyright $\odot 2018$ by authors and Open Access Library Inc.

This work is licensed under the Creative Commons Attribution International License (CC BY 4.0).

http://creativecommons.org/licenses/by/4.0/

\section{cc) (1) Open Access}

\begin{abstract}
A new PLSGP (potential smokers-light smokers-persistent smokers-giving up smokers-potential smokers) model with birth and death rates on complex heterogeneous networks is presented. Using the mean-field theory, we obtain the basic reproduction number $R_{0}$ and find that basic reproduction number for constant contact is independent of the topology of the underlying networks. When $R_{0}<1$, the smoking-free equilibrium is globally asymptotically stable, then the smoking will disappear. When $R_{0}>1$, the smoking-present equilibrium is global attractivity, then the number of smoker will remain stable and smoking will become endemic. Numerical simulations illustrated theoretical results. Our result shows that the model is very important to control the spread of the smoking.
\end{abstract}

\section{Subject Areas}

Complex Network Models, Dynamical System, Network Modeling and Simulation, Numerical Mathematics, Numerical Methods

\section{Keywords}

Spreading Dynamic, Smoking, Basic Reproduction Number, Equilibrium, Scale-Free Network

\section{Introduction}

Smoking is closely related to health, and smoking ranks fourth among the top 10 risk factors for health, according to the World Health Organization report. Tobacco has been identified as a primary carcinogen around the world. Smokers are 10 to 30 times more likely to develop lung cancer than non-smokers. Smoking problem of people has become a significant public health concern. The beha- 
vior of smoking often causes a range of negative consequences. Long-term smoking produces negative changes in the heart, such as heart rate and blood pressure rise. Smoking damages almost all parts of the human body and contributes to a number of human diseases including lung cancer, respiratory disease, heart disease, alimentary canal effect and eventually death. Due to the increasing in the number of smokers, tobacco use is also as a disease to be treated.

In recent years, many types of epidemic models are discussed, such as virus dynamics models [1] [2], tuberculosis models [3] [4], and HIV models [5] [6]. One of the famous representative works in this area was done by Pastor-Satorras and Vespignani [7]. They presented a detailed analytical and numerical study on an SIS epidemic model in the highly heterogeneous networks (i.e., scale-free networks). The most striking result is that they found the absence of the epidemic threshold in these networks. That is, the threshold approaches zero in the limit of a large number of edges and nodes, and even a quite small infectious rate can produce a major epidemic outbreak. Liu XD et al. [8] presented an SIS (susceptible-infected-susceptible) epidemic model with infective medium and feedback mechanism on scale-free networks. They found that the epidemic is not only spread between individuals by direct contacts but also transmitted by medium, people's initial response when epidemic disease outbreaks have been also considered. Moreno et al. [9] also found these similar conclusions for an $S I R$ (susceptible-infected-removed) epidemic model on scale-free networks. The epidemic model is constantly evolving, such as SIRS (susceptible-infected-removedsusceptible), SEIR (susceptible-infected-exposed-removed), SIQRS (susceptible-infected-quarantined-recovered-susceptible) [10] [11] [12] [13] [14].

Studying of smoking behavior has attracted the attention of many scholars and researchers recently. In order to explore the spread rule of smoking, some models are developed. Castillo-Garsow et al. [15] proposed a mathematical model for giving up smoking in the first time. In this model, a total constant population was divided into three states: potential smokers, that is, people who do not smoke yet but might become smokers in the future $(P)$, smokers $(S)$, and quit smokers $(Q)$. Zaman [16] extended the work of Castillo-Garsow et al. [15] by adding the population of occasional smokers in the model, and presented qualitative behavior of the model. Zaman [17] presented the optimal campaigns in the smoking dynamics. They consider two possible control variables in the form of education and treatment campaigns oriented to decrease the attitude towards smoking and first showed the existence of an optimal control for the control problem.

However, in real life, some potential smokers may become light smoker since they contact with light smokers or persistent smokers. Some quit smokers may be only temporary quit smokers, so they will become potential smokers again. Enlightening by the previously mentioned cases, we present a PLSGP giving up smoking model on scale-free network. The paper is organized as follows: The model is formulated in Section 2. The basic reproduction number and existence 
of smoking equilibriums are calculated in Section 3. In Section 4, we analyze the stability of the equilibria. In Section 5, sensitivity analysis and numerical simulations are illustrated. In Section 6, we give some conclusions and discussions.

\section{The Model Formulation}

In this paper, we establish the giving up smoking model as Figure 1. From Figure 1, the total population is divided into four compartments, namely, the potential smokers compartment $(P)$, light or occasion smokers compartment $(L)$, persistent smokers compartment $(S)$, and quit smokers group $(G)$. The total recruitment number into this homogeneous social mixing community is $b$. Transmission coefficient from the potential smokers compartment to the light smokers compartment is $\rho_{1}$, Transmission coefficient from the potential smokers compartment to the persistent smokers compartment is $\rho_{2}$, Transmission coefficient from the light smokers compartment to the persistent smokers compartment is $\alpha$, The permanent quit smoking rate is $\beta$, The relapse rate of which temporary quit people to become potential smokers is $\delta$. Naturally death rate is $\mu$. The total population size is $N(t)$. Let $P_{k}(t), L_{k}(t), S_{k}(t)$, $G_{k}(t)$ be the relative densities of potential smokers, light smokers, persistent smokers and quit smokers nodes of degree $k$ at time $t$, respectively.

With these assume, the dynamic mean-field equations of the PLSGP model can be written as follows:

$$
\left\{\begin{array}{l}
\frac{\mathrm{d} P_{k}(t)}{\mathrm{d} t}=b+\delta G_{k}(t)-k\left(\rho_{1} \Theta_{1}+\rho_{2} \Theta_{2}\right) P_{k}(t)-\mu P_{k}(t) \\
\frac{\mathrm{d} L_{k}(t)}{\mathrm{d} t}=k\left(\rho_{1} \Theta_{1}+\rho_{2} \Theta_{2}\right) P_{k}(t)-(\alpha+\mu+\gamma) L_{k}(t) \\
\frac{\mathrm{d} S_{k}(t)}{\mathrm{d} t}=\alpha L_{k}(t)-(\mu+\beta) S_{k}(t) \\
\frac{\mathrm{d} G_{k}(t)}{\mathrm{d} t}=\beta S_{k}(t)+\gamma L_{k}(t)-(\mu+\delta) G_{k}(t)
\end{array}\right.
$$

where

$$
\begin{aligned}
& \Theta_{1}(t)=\sum_{i} P(i \mid k) L_{i}(t)=\langle k\rangle^{-1} \sum_{i} i P(i) L_{i}(t) \\
& \Theta_{2}(t)=\sum_{i} P(i \mid k) S_{i}(t)=\langle k\rangle^{-1} \sum_{i} i P(i) S_{i}(t)
\end{aligned}
$$

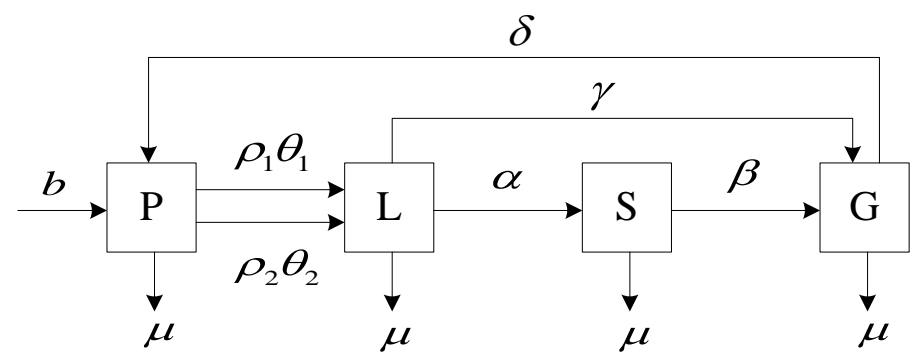

Figure 1. Transfer diagram for somking model. 
where, $P(k)>0$ is the probability that a node has degree $k$ and thus $\sum_{k=1}^{n} P(k)=1,\langle k\rangle=\sum_{k=1}^{n} k P(k)$ denotes the average degree. $\rho=\rho_{1} \Theta_{1}+\rho_{2} \Theta_{2}$. Clearly, these variables obey the normalization condition:

$$
P_{k}(t)+L_{k}(t)+S_{k}(t)+G_{k}(t)=1 .
$$

The initial conditions for system can be given as follows

$S_{k}(0)=1-P_{k}(0)-L_{k}(0)-G_{k}(0) \geq 0, \quad R_{k}(0) \geq 0, \quad S_{k}(0) \geq 0, \quad C_{k}(0) \geq 0$. In this model, we assumed $\mu$ equal to $p$.

$$
\left\{\begin{array}{l}
\frac{\mathrm{d} L_{k}(t)}{\mathrm{d} t}=k \rho\left(1-L_{k}(t)-S_{k}(t)-G_{k}(t)\right) P_{k}(t)-(\alpha+\mu+\gamma) L_{k}(t) \\
\frac{\mathrm{d} S_{k}(t)}{\mathrm{d} t}=\alpha L_{k}(t)-(\mu+\beta) S_{k}(t) \\
\frac{\mathrm{d} G_{k}(t)}{\mathrm{d} t}=\beta S_{k}(t)+\gamma L_{k}(t)-(\mu+\delta) G_{k}(t)
\end{array}\right.
$$

\section{The Basic Reproduction Number and Equilibrium}

Theorem 1. Consider system (2.1). Define $R_{0}=\frac{\left\langle k^{2}\right\rangle\left(\rho_{1}(\beta+\mu)+\rho_{2} \alpha\right)}{\langle k\rangle(\beta+\mu)(\alpha+\mu+\gamma))}$. There always exists the smoking-free equilibrium $E_{0}(1,0,0,0)$. When $R_{0}>1$, the system has an occasion smoking equilibrium $E^{*}\left(P_{k}^{*}, L_{k}^{*}, S_{k}^{*}, G_{k}^{*}\right)$.

Proof. To get the information-prevailing equilibrium solution $E^{*}\left(P_{k}^{*}, L_{k}^{*}, S_{k}^{*}, G_{k}^{*}\right)$, we need to make the right side of system equal to zero, it should satisfy

$$
\left\{\begin{array}{l}
b+\delta G_{k}^{*}(t)-k \rho^{*} P_{k}^{*}(t)-\mu P_{k}^{*}(t)=0 \\
k \rho^{*} P_{k}^{*}(t)-(\alpha+\mu+\gamma) L_{k}^{*}(t)=0 \\
\alpha L_{k}^{*}(t)-(\mu+\beta) S_{k}^{*}(t)=0 \\
\beta S_{k}^{*}(t)+\gamma L_{k}^{*}(t)-(\mu+\delta) G_{k}^{*}(t)=0
\end{array}\right.
$$

where $\rho^{*}=\rho_{1} \Theta_{1}^{*}+\rho_{2} \Theta_{2}^{*}$, we follow from (3.1) that

$$
\begin{aligned}
& S_{k}^{*}(t)=\frac{k \rho \alpha(\mu+\delta)}{(\mu+\delta)(\mu+\beta)(\mu+\alpha+\gamma)+k \rho[(\mu+\beta)(1+\gamma)+\alpha(\beta+(\mu+\delta))]} \\
& L_{k}^{*}(t)=\frac{k \rho(\mu+\delta)(\mu+\beta)}{(\mu+\delta)(\mu+\beta)(\mu+\alpha+\gamma)+k \rho[(\mu+\beta)(1+\gamma)+\alpha(\beta+(\mu+\delta))]}
\end{aligned}
$$

Obviously, $\rho^{*}=0$ satisfies (3.1). Hence, $P_{k}=1$ and $L_{k}=S_{k}=R_{k}=0$ is an equilibrium of (2.1), which is called the smoking-free equilibrium.

Substituting $L_{k}^{*}$ and $S_{k}^{*}$ of (3.2) into $\rho^{*}$

Let $\rho^{*} \cong f\left(\rho^{*}\right)$

Clearly, $\rho^{*}=0$ is a solution of equation. To ensure the equation has a nontrivial solution, the following condition must should satisfied 


$$
\left.\frac{\mathrm{d} f\left(\rho^{*}\right)}{\mathrm{d} \rho^{*}}\right|_{\rho^{*}}=\frac{\partial f\left(\rho^{*}\right)}{\partial L_{k}^{*}}+\frac{\partial f\left(\rho^{*}\right)}{\partial S_{k}^{*}}>1 \text { and } f(1) \leq 1 .
$$

We can obtain the reproductive number

$$
R_{0}=\frac{\left\langle k^{2}\right\rangle\left(\rho_{1}(\beta+\mu)+\rho_{2} \alpha\right)}{\langle k\rangle(\beta+\mu)(\alpha+\mu+\gamma)} .
$$

\section{Stability Analysis of the Equilibrium}

Theorem 2. When $R_{0}<1$, the smoking-free equilibrium of system (2.1) is globally asymptotically stable.

Proof. The Jacobian matrix of the smoking-free equilibrium of system (2.1), which is a $3 n \times 3 n$ matrix, can be written as follows:

$$
J=\left(\begin{array}{ccc}
A_{11} & \cdots & A_{1 n} \\
\vdots & \ddots & \vdots \\
A_{n 1} & \cdots & A_{n n}
\end{array}\right)
$$

where

$$
\begin{gathered}
A_{11}=\left(\begin{array}{ccc}
-\delta-\mu & -\delta-\rho_{1} P_{1} & -\delta-\rho_{2} P_{1} \\
0 & \rho_{1} P_{1}-(\alpha+\mu+\gamma) & \rho_{2} P_{1} \\
0 & \alpha & -\mu-\beta
\end{array}\right) \\
A_{1 n}=\left(\begin{array}{ccc}
0 & -\rho_{1} P_{n} & -\rho_{2} P_{n} \\
0 & \rho_{1} P_{n} & \rho_{2} P_{n} \\
0 & 0 & 0
\end{array}\right) \\
A_{1 n}=\left(\begin{array}{ccc}
0 & -n \rho_{1} P_{n} & -n \rho_{2} P_{n} \\
0 & n \rho_{1} P_{n} & n \rho_{2} P_{n} \\
0 & 0 & 0
\end{array}\right) \\
A_{n n}=\left(\begin{array}{ccc}
-\delta-\mu & -\delta-n \rho_{1} P_{n} & -\delta-n \rho_{2} P_{n} \\
0 & n \rho_{1} P_{n}-(\mu+\alpha+\gamma) & n \rho_{2} P_{n} \\
0 & \alpha & -\mu-\beta
\end{array}\right)
\end{gathered}
$$

A direct calculation leads to the characteristic polynomial of the smoking-free equilibrium in the following from:

$$
(\lambda+(\mu+\alpha+\gamma))^{n-1}(\lambda+\mu+\beta)\left(\lambda^{2}+p \lambda+q\right)=0,
$$

where $p=(\mu+\beta)+(\mu+\alpha+\gamma)-\rho_{1} \sum_{i=1}^{n} i P(i)$ and $q=(\mu+\beta)\left(\mu+\alpha+\gamma+\rho_{1}\right)-\alpha \rho_{2} \sum_{i=1}^{n} i P(i)$.

Note that $R_{0}<1$ is equivalent to $q>0$ and that $R_{0}<1$ also implies: $(\mu+\beta)+(\mu+\alpha+\gamma)>\rho_{1} \sum_{i=1}^{n} i P(i)$, which means $p>0$. Note that $R_{0}<1$ is equivalent to $q>0$ and that $R_{0}<1$ also implies and, which means $p>0$. Therefore, there exists a unique positive eigenvalue $\lambda$ of $J$ if and only if $R_{0}>1$, otherwise, if $R_{0}<1$, all real-valued eigenvalues of $J$ are negative. By the Perron-Frobenius theorem, it implies that the maximal real part of all eigen- 
values of $J$ is positive if and only if $R_{0}>1$. Then, a theorem of Lajmanovich and York [18] yields the results of this theorem. The proof is thus completed.

Next, the globally attractivity of positive endemic equilibrium is discussed. The main result is given in the following theorem.

Lemma 1. [19] if $a>0, b>0$ and $\frac{\mathrm{d} x(t)}{\mathrm{d} t} \geq b-a x$, when $t \geq 0$ and $x(0) \geq 0$, we have $t \geq 0 \lim _{t \rightarrow \infty}$ inf $x(t) \geq \frac{b}{a}$, if $a>0, b>0$ and $\frac{\mathrm{d} x(t)}{\mathrm{d} t} \leq b-a x$, when $t \geq 0$ and $x(0) \geq 0$, we have $\lim _{t \rightarrow \infty} \sup x(t) \leq \frac{b}{a}$.

Theorems 3. Suppose that $\left(L_{k}(t), S_{k}(t), G_{k}(t)\right)$ is a solution of (2.4), with $L_{k}(0)>0, S_{k}(0)>0, G_{k}(0)>0$ and $R_{0}>1$. If $R_{0}>1$, then $\lim _{t \rightarrow \infty}\left(L_{k}(t), S_{k}(t), G_{k}(t)\right)=\left(L_{k}^{*}(t), S_{k}^{*}(t), G_{k}^{*}(t)\right)$, where $\left(L_{k}^{*}(t), S_{k}^{*}(t), G_{k}^{*}(t)\right)$ is the unique smoking equilibrium of (2.4) for $k=1,2, \cdots, n$.

Proof: In the following, $k$ is fixed to be any integer in $(1,2, \cdots, n)$. There exists a sufficiently small constant $\xi(0<\xi<1)$ and a larger enough constant $T>0$ such that $L_{k}(t) \geq \xi$ and $S_{k}(t) \geq \xi$ for $t>T$, therefore $\left(\rho_{1}+\rho_{2}\right) \xi<\rho(t)<\rho_{1}+\rho_{2}$ for $t>T$. Submit this into the first equation of (2.4) gives

$$
L_{k}^{\prime}(t) \leq k\left(\rho_{1}+\rho_{2}\right)\left(1-L_{k}(t)\right)-(\alpha+\mu+\gamma) L_{k}(t), t>T
$$

By Lemma 1 , for any given constant $0<\xi<\frac{\mu+\alpha+\gamma}{k\left(\rho_{1}+\rho_{2}\right)(\mu+\alpha+\gamma)}$, there exists a $t_{1}>T$, such that $L_{k}(t) \leq X_{k}^{(1)}+\xi_{1}$ for $t>t_{1}$, where

$$
L_{k}(t) \leq X_{k}^{(1)}+\xi_{1}=\frac{k\left(\rho_{1}+\rho_{2}\right)}{k\left(\rho_{1}+\rho_{2}\right)+(\alpha+\mu+\gamma)}+\xi_{1}<1, t>t_{1}
$$

From the second equation of (2.4), it follows that

$$
S_{k}^{\prime}(t) \leq \alpha\left(1-S_{k}(t)\right)-(\mu+\beta) S_{k}(t), t>t_{1}
$$

Hence, for any given constant $0<\xi_{2}<\min \left\{1 / 2, \xi_{1},(\beta+\mu)(\mu+\alpha+\beta)^{-1}\right\}$, there exists a $t_{2}>t_{1}$, such that $S_{k}(t) \leq Y_{k}^{(1)}-\xi_{2}$ for $t>t_{2}$, where

$$
S_{k}(t) \leq Y_{k}^{(1)}+\xi_{2}<\alpha(\alpha+\mu+\beta)^{-1}+\xi_{2}<1, t>t_{2}
$$

Then, it follows from the third equation of (2.4),

$$
G_{k}^{\prime}(t) \leq \beta\left(1-G_{k}(t)\right)+\gamma\left(1-G_{k}(t)\right)-(\mu+\delta) G_{k}(t), t>t_{2}
$$

Similarly, for any given constant

$0<\xi_{3}<\min \left\{1 / 3, \xi_{2},(\delta+\mu)(\mu+\delta+\beta+\gamma)^{-1}+\xi_{3}\right\}$, there exists a $t_{3}>t_{2}$, such that $G_{k}(t) \leq Z_{k}^{(1)}+\xi_{3}$ for $t>t_{3}$, where

$$
G_{k}(t) \leq Z_{k}^{(1)}+\xi_{3}<(\beta+\gamma)(\mu+\beta+\gamma+\delta)^{-1}+\xi_{3}<1, t>t_{3}
$$

Since $\left(\rho_{1}+\rho_{2}\right) \xi<\rho(t)$, we substitute this into the first equation of (2.4) 


$$
L_{k}^{\prime}(t) \geq k \xi\left(\rho_{1}+\rho_{2}\right)\left(1-L_{k}(t)-Y_{k}^{(1)}-Z_{k}^{(1)}\right)-(\alpha+\mu+\gamma) L_{k}(t), t>T
$$

So for any given enough small constant

$0<\xi_{4}<\min \left\{1 / 4, \xi_{3}, \frac{k \xi\left(\rho_{1}+\rho_{2}\right)\left(1-L_{k}(t)-Y_{k}^{(1)}-Z_{k}^{(1)}\right)}{k \xi\left(\rho_{1}+\rho_{2}\right)+(\alpha+\mu+\gamma)}\right\}$, there exists a $t_{4}>t_{3}$, such that $L_{k}(t) \geq x_{k}^{(1)}-\xi_{4}$ for $t>t_{4}$, where

$$
L_{k}(t) \geq x_{k}^{(1)}-\xi_{4}=\frac{k \xi\left(\rho_{1}+\rho_{2}\right)\left(1-L_{k}(t)-Y_{k}^{(1)}-Z_{k}^{(1)}\right)}{k \xi\left(\rho_{1}+\rho_{2}\right)+(\alpha+\mu+\gamma)}-\xi_{4}, t>t_{4}
$$

It follows that

$$
S_{k}^{\prime}(t) \geq \alpha x_{k}^{(1)}-(\mu+\eta) S_{k}(t), t>t_{4}
$$

So for any given enough small constant $0<\xi_{5}<\min \left\{1 / 5, \xi_{4}, \alpha x_{k}^{(1)}[(\mu+\beta)]^{-1}\right\}$, there exists a $t_{5}>t_{4}$, such that $S_{k}^{\prime}(t) \geq y_{k}^{(1)}-\xi_{5}$ for $t>t_{5}$, where

$$
S_{k}^{\prime}(t) \geq y_{k}^{(1)}-\xi_{5}=\alpha x_{k}^{(1)}(\mu+\beta)^{-1}-\xi_{5}, t>t_{5}
$$

From the third equation of (2.1) implies that

$$
G_{k}^{\prime}(t) \geq \beta y_{k}^{(1)}+\gamma x_{k}^{(1)}-(\mu+\delta) G_{k}(t), t>t_{5}
$$

So for any given enough small constant

$0<\xi_{6}<\min \left\{1 / 6, \xi_{5},\left[\beta y_{k}^{(1)}+\gamma x_{k}^{(1)}\right](\mu+\delta)^{-1}\right\}$, there exists a $t_{6}>t_{5}$, such that $G_{k}(t) \geq z_{k}^{(1)}-\xi_{6}$ for $t>t_{6}$, where

$$
G_{k}(t) \geq z_{k}^{(1)}-\xi_{6}=\left(\beta y_{k}^{(1)}+\gamma x_{k}^{(1)}\right)(\mu+\delta)^{-1}, t>t_{6}
$$

Due to $\xi$ is a small positive constant, we can derive that $0<x_{k}^{(1)} \leq X_{k}^{(1)}<1$, $0<y_{k}^{(1)} \leq Y_{k}^{(1)}<1$ and $0<z_{k}^{(1)} \leq Z_{k}^{(1)}<1$. Let

$$
\begin{aligned}
& q^{(j)}=\frac{1}{\langle k\rangle} \sum_{j=1}^{n} P(i)\left(\rho_{1} x_{i}^{(j)}+\rho_{2} y_{i}^{(j)}\right), \\
& Q^{(j)}=\frac{1}{\langle k\rangle} \sum_{j=1}^{n} P(i)\left(\rho_{1} X_{i}^{(j)}+\rho_{2} Y_{i}^{(j)}\right), \quad j=1,2, \cdots
\end{aligned}
$$

We can easily get $0<q^{(j)} \leq \rho(t) \leq Q^{(j)}<\rho_{1}+\rho_{2}, \quad t>t_{6}$.

Again, from the first equation of (2.1), it has

$$
L_{k}^{\prime}(t) \leq k Q^{(1)}\left(1-L_{k}(t)-y_{k}^{(1)}-z_{k}^{(1)}\right)-(\mu+\alpha+\gamma), t>t_{6}
$$

Hence, for any given constant $0<\xi_{7}<\min \left\{1 / 7, \xi_{6}\right\}$, there exists a $t_{7}>t_{6}$, such that

$$
\begin{aligned}
& I_{k}(t) \leq X_{k}^{(2)} \\
& =\min \left\{X_{k}^{(1)}+\xi_{1}, k Q^{(1)}\left(1-y_{k}^{(1)}-z_{k}^{(1)}\right)\left[k Q^{(1)}+\mu+\alpha+\gamma\right]^{-1}+\xi_{7}\right\}, t>t_{7}
\end{aligned}
$$

Then, from the second equation of (2.1), we have 


$$
S_{k}^{\prime}(t) \leq Y_{k}^{(2)}-(\mu+\beta) S_{k}(t), t>t_{7}
$$

So, for any given constant $0<\xi_{8}<\min \left\{1 / 8, \xi_{7}\right\}$, there exists a $t_{8}>t_{7}$, such that

$$
S_{k}(t) \leq Y_{k}^{(2)}=\min \left\{Y_{k}^{(2)}+\xi_{2}, \alpha X_{k}^{(2)}(\mu+\beta)^{-1}+\xi_{8}\right\}, t>t_{8}
$$

Consequently, from the third equation of (2.1), we have

$$
G_{k}^{\prime}(t) \leq \beta Y_{k}^{(2)}+\gamma X_{k}^{(1)}-(\mu+\delta) G_{k}(t), t>t_{8}
$$

Hence, for any given constant $0<\xi_{9}<\min \left\{1 / 9, \xi_{8}\right\}$, there exists a $t_{8}>t_{7}$, such that

$$
G_{k}(t) \leq Z_{k}^{(2)}=\min \left\{Z_{k}^{(1)}-\xi_{3},\left(\beta Y_{k}^{(2)}+\gamma X_{k}^{(2)}\right)(\delta+\mu)^{-1}+\xi_{9}\right\}, t_{9}>t_{8}
$$

Turning back, one has

$$
L_{k}^{\prime}(t) \geq k q^{(1)}\left(1-L_{k}(t)-Y_{k}^{(1)}-Z_{k}^{(1)}\right)-(\alpha+\mu+\gamma) L_{k}(t), t>t_{9}
$$

So, for any given enough small constant $0<\xi_{10}<\min \left\{1 / 10, \xi_{9}\right\}$, there exists a $t_{10}>t_{9}$, such that $G_{k}(t) \geq x_{k}^{(2)}+\xi_{10}$ for $t>t_{10}$, where

$$
\begin{gathered}
L_{k}(t) \geq x_{k}^{(2)}=\max \left\{x_{k}^{(1)}-\xi_{4}, k q^{(1)}\left(1-Y_{k}^{(1)}-Z_{k}^{(1)}\right)\left(k q^{(1)}+(\alpha+\mu+\gamma)\right)^{-1}\right\} \\
S_{k}^{\prime}(t) \geq \alpha x_{k}^{(2)}-(\mu+\beta) S_{k}(t), t>t_{10}
\end{gathered}
$$

So for any given enough small constant $0<\xi_{11}<\min \left\{1 / 11, \xi_{10}\right\}$, there exists a $t_{11}>t_{10}$, such that $S_{k}(t) \geq y_{k}^{(2)}+\xi_{11}$ for $t>t_{10}$, where

$$
S_{k}(t) \geq y_{k}^{(2)}=\max \left\{y_{k}^{(1)}-\xi_{5}, \alpha x_{k}^{(2)}(\gamma+\mu)+\xi_{11}\right\}, t>t_{11}
$$

From the third equation of (2.1) implies that

$$
G_{k}^{\prime}(t) \geq \beta y_{k}^{(2)}+\gamma x_{k}^{(2)}-(\mu+\delta) G_{k}(t)
$$

So, for any given enough small constant $0<\xi_{12}<\min \left\{1 / 12, \xi_{11}\right\}$, there exists a $t_{12}>t_{11}$, such that $G_{k}(t) \geq z_{k}^{(2)}+\xi_{12}$ for $t>t_{12}$, where

$$
G_{k}(t) \geq z_{k}^{(2)}=\max \left\{z_{k}^{(1)}-\xi_{6},\left[\left(\beta y_{k}^{(2)}+\gamma x_{k}^{(2)}\right)(\mu+\delta)^{-1}+\xi_{12}\right](\mu+\delta)^{-1}\right\}
$$

Repeating the above analyses and calculation, we get six sequences $X_{k}^{(i)}, Y_{k}^{(i)}, Z_{k}^{(i)}, x_{k}^{(i)}, y_{k}^{(i)}, z_{k}^{(i)}, \quad i=1,2, \cdots$. Due to the first three are monotone decreasing sequences and the last three are monotone increasing, there exists a sufficiently large positive integer $L \geq 2$, such that $l \geq L$ :

$$
\begin{gathered}
X_{k}^{(l)}=\frac{k Q^{(l-1)}\left(1-y_{k}^{(l-1)}-z_{k}^{(l-1)}\right)}{k Q^{(l-1)}+\mu+\alpha+\gamma}+\xi_{6 l-5}, \quad Y_{k}^{(l)}=\frac{\alpha X_{k}^{(l)}}{\mu+\beta}+\xi_{6 l-4}, \\
Z_{k}^{(l)}=\frac{\beta Y_{k}^{(l)}+\gamma X_{k}^{(l)}}{\delta+\mu}+\xi_{6 l-3}, \quad x_{k}^{(l)}=\frac{k q^{(l-1)}\left(1-Y_{k}^{(l-1)}-Z_{k}^{(l-1)}\right)}{k q^{(l-1)}+\mu+\alpha+\gamma}+\xi_{6 l-2}, \\
y_{k}^{(l)}=\frac{\alpha x_{k}^{(l)}}{\mu+\beta}+\xi_{6 l-1}, \quad z_{k}^{(l)}=\frac{\beta y_{k}^{(l)}+\gamma x_{k}^{(l)}}{\delta+\mu}+\xi_{6 l} .
\end{gathered}
$$


We can easy get that

$$
x_{k}^{(l)} \leq L_{k}(t) \leq X_{k}^{(l)}, y_{k}^{(l)} \leq S_{k}(t) \leq Y_{k}^{(l)}, z_{k}^{(l)} \leq G_{k}(t) \leq Z_{k}^{(l)}, t>t_{6 l}
$$

Since the sequential limits of (4.26) exist, let $\lim _{l \rightarrow \infty} \Delta_{k}^{(l)}=\Delta_{k}$, where $\Delta_{k}^{(l)} \in\left\{X_{k}^{(l)}, Y_{k}^{(l)}, Z_{k}^{(l)}, x_{k}^{(l)}, y_{k}^{(l)}, z_{k}^{(l)}, Q_{k}^{(l)}, q_{k}^{(l)}\right\}$ and $\Delta_{k} \in\left\{X_{k}, Y_{k}, Z_{k}, x_{k}, y_{k}, z_{k}, Q_{k}, q_{k}\right\}$,

Noting that $0<\xi_{1}<1 / l$, one has $\xi_{1} \rightarrow 0$ as $l \rightarrow \infty$. In the six sequences of (4.26), by taking $l \rightarrow \infty$, it follows from (4.26) that

$$
\begin{gathered}
X_{k}=\frac{k Q\left(1-y_{k}-z_{k}\right)}{k Q+\mu+\alpha+\gamma}, \quad Y_{k}=\frac{\alpha X_{k}}{\mu+\beta}, \quad Z_{k}=\frac{\beta Y_{k}+\gamma X_{k}}{\delta+\mu}, \\
x_{k}=\frac{k q\left(1-Y_{k}-Z_{k}\right)}{k q+\mu+\alpha+\gamma}, \quad y_{k}=\frac{\alpha x_{k}}{\mu+\beta}, \quad Z_{k}=\frac{\beta y_{k}+\gamma X_{k}}{\delta+\mu} . \\
q^{(j)}=\frac{1}{\langle k\rangle} \sum_{j=1}^{n} P(i)\left(\rho_{1} x_{i}^{(j)}+\rho_{2} y_{i}^{(j)}\right), Q^{(j)}=\frac{1}{\langle k\rangle} \sum_{j=1}^{n} P(i)\left(\rho_{1} X_{i}^{(j)}+\rho_{2} Y_{i}^{(j)}\right),
\end{gathered}
$$

Substituting (4.27) and (4.28) into $q$ and $Q$, respectively, one has

$$
\begin{aligned}
1= & \left(\rho_{1}+\rho_{1} \frac{\alpha}{\mu+\beta}\right) \sum_{i=1}^{n} \frac{i P(i)}{M_{i}}\left[Q(\delta+\mu)^{2}(\beta+\mu)^{2}(i q+\mu+\gamma+\alpha)\right. \\
& -i q Q H(\delta+\mu)(\beta+\mu)] \\
1= & \left(\rho_{1}+\rho_{1} \frac{\alpha}{\mu+\beta}\right) \sum_{i=1}^{n} \frac{i P(i)}{G_{i}}\left[q(\delta+\mu)^{2}(\beta+\mu)^{2}(i Q+\mu+\gamma+\alpha) .\right. \\
& -i q Q H(\delta+\mu)(\beta+\mu)]
\end{aligned}
$$

where

$$
\begin{gathered}
M_{i}=\left[(\delta+\mu)^{2}(\beta+\mu)^{2}(i q+\mu+\gamma+\alpha)(i Q+\mu+\gamma+\alpha)-i^{2} q Q H^{2}\right], \\
H=\alpha(\delta+\mu)(\mu+\gamma+\alpha)+\gamma(\beta+\mu)(\mu+\gamma+\alpha)+\alpha \beta(\mu+\gamma+\alpha) .
\end{gathered}
$$

By subtracting the above two equations, it arrives at

$$
\begin{aligned}
0= & (Q-q)(\delta+\mu)(\beta+\mu)\left(\rho_{1}+\rho_{1} \frac{\alpha}{\mu+\beta}\right) \\
& \times \sum_{i=1}^{n} \frac{i^{2} P(i)}{G_{i}}[(\mu+\gamma+\alpha)(\delta+\mu)(\mu+\sigma)-H] .
\end{aligned}
$$

It is obviously that $q=Q$, so $\frac{1}{\langle k\rangle} \sum_{i=1}^{n} i P(i)\left(\rho_{1}\left(X_{i}-x_{i}\right)+\rho_{2}\left(Y_{i}-y_{i}\right)\right)=0$, which sees that $X_{i}=x_{i}, Y_{i}=y_{i}$, for $i=1,2, \cdots, n$. From (4.26) and (4.27), it follows that

$$
\lim _{t \rightarrow \infty} L_{k}(t)=X_{k}=x_{k}, \lim _{t \rightarrow \infty} S_{k}(t)=Y_{k}=y_{k}, \quad \lim _{t \rightarrow \infty} G_{k}(t)=Z_{k}=z_{k} .
$$

Finally, substituting $q=Q$ into (4.26), in view of (3.2) and (4.28), it obtains $L_{k}=L_{k}^{*}, S_{k}=S_{k}^{*}$, and $G_{k}=G_{k}^{*}$. The proof is completed. 


\section{Numerical Simulations}

In this section, some sensitivity analyses are presented to illustrate the result of the smoking model (2.1). We consider the system (2.1) on a scale-free network with the degree distribution $P(k)=\xi k^{-r}, 2<r \leq 3$ and $\sum_{i=1} P(i)=1$. Consider system (2.1) with $r=3$ and $n=1000$.

Parameters used in the simulations list as follows: in Figure 2, we choose $\alpha=0.25, \quad b=0.2, \mu=0.2, \gamma=0.6, \beta=0.5, \rho_{1}=0.15, \rho_{2}=0.2, \delta=0.15$, thus the threshold value $R_{0}=0.708<1$. The figure show that when $R_{0}<1, L_{k}$ and $S_{k}$ approach to zero, the smoking population will ultimately disappear, which means that smoking will disappear. In Figure 3, we choose $\alpha=0.2$, $b=0.2, \quad \mu=0.2, \gamma=0.2, \quad \beta=0.5, \rho_{1}=0.5, \rho_{2}=0.2, \delta=0.1$, thus the threshold value $R_{0}=4.18>1$. The figure show that when $R_{0}>1, L_{k}$ and $S_{k}$ maintain at a positive stationary level, which means that the smoking become endemic "disease".

Figure 4 and Figure 5 show the dynamic behavior of light problem smoking and heavier problem smoking with different degree when $R_{0}<1$. Figure 6 and Figure 7 show the dynamic behavior of light problem smoking and heavier problem smoking with different degree when $R_{0}>1$. We find that the larger degree leads to larger value of the smoking level.

\section{Conclusion}

In this paper, we propose a PLSGP giving up smoking model on scale-free network.

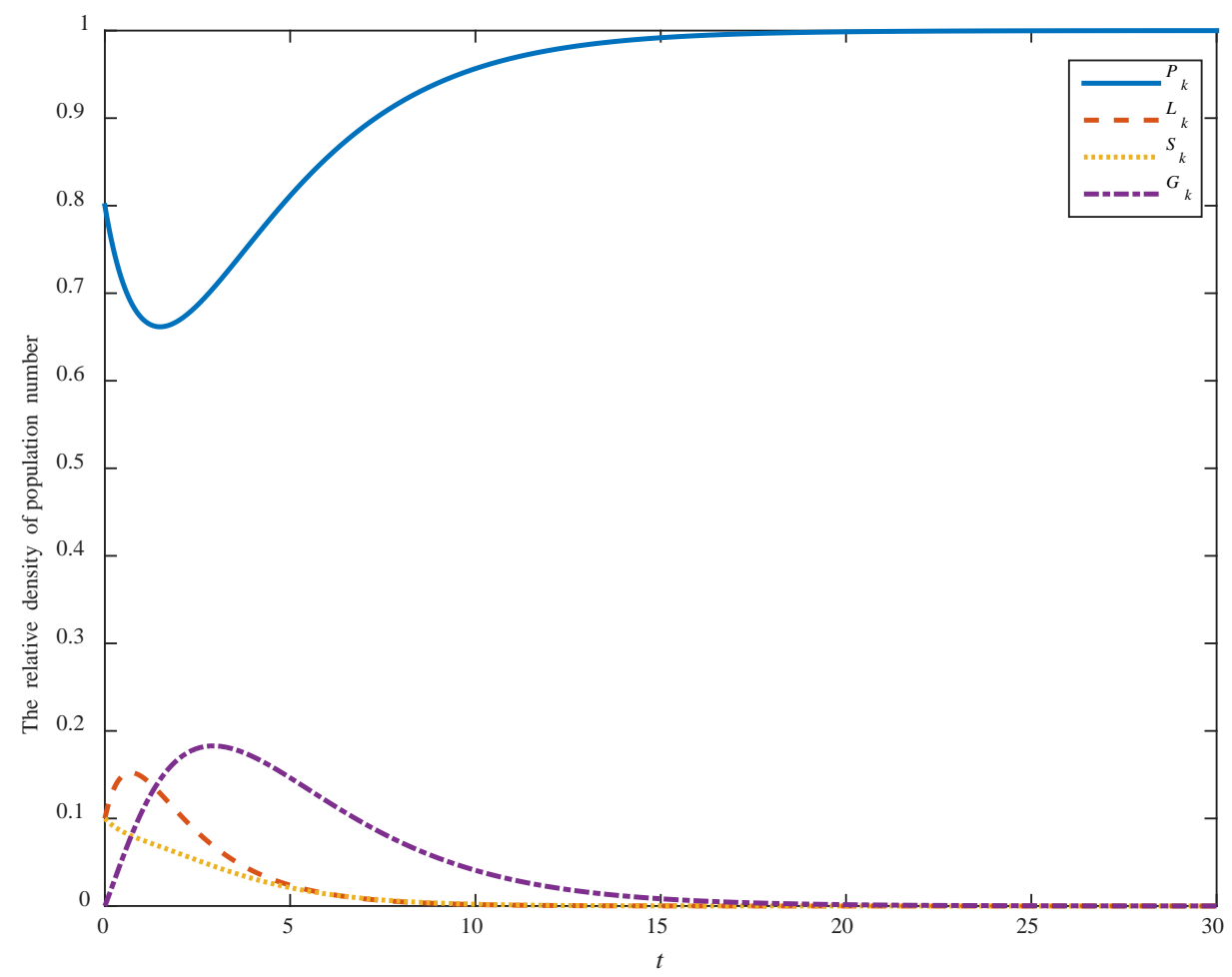

Figure 2. The relative density of population number when $R_{0}<1$. 


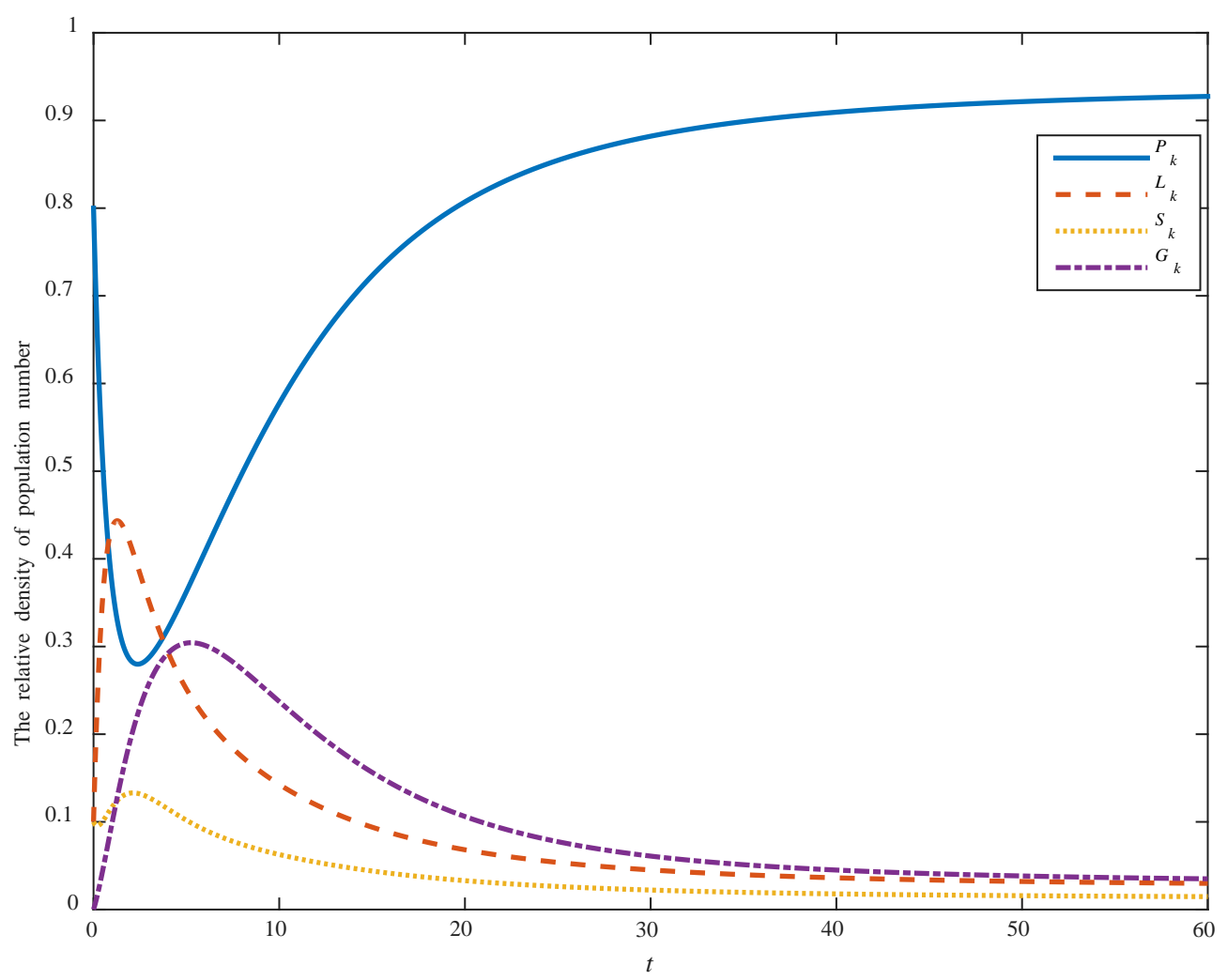

Figure 3. The relative density of population number when $R_{0}>1$.

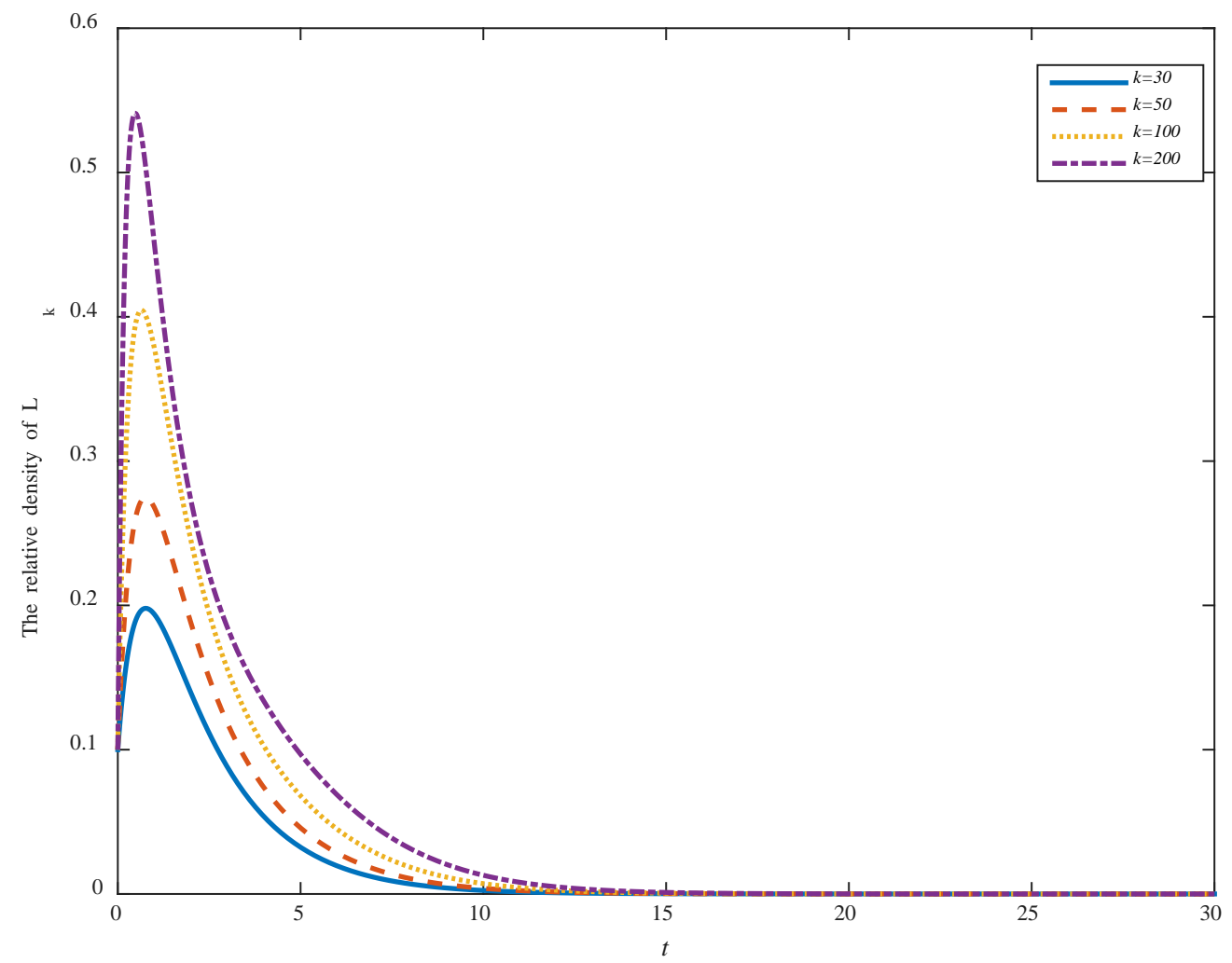

Figure 4. Dynamic behavior of light smoker with different degree when $R_{0}<1$. 


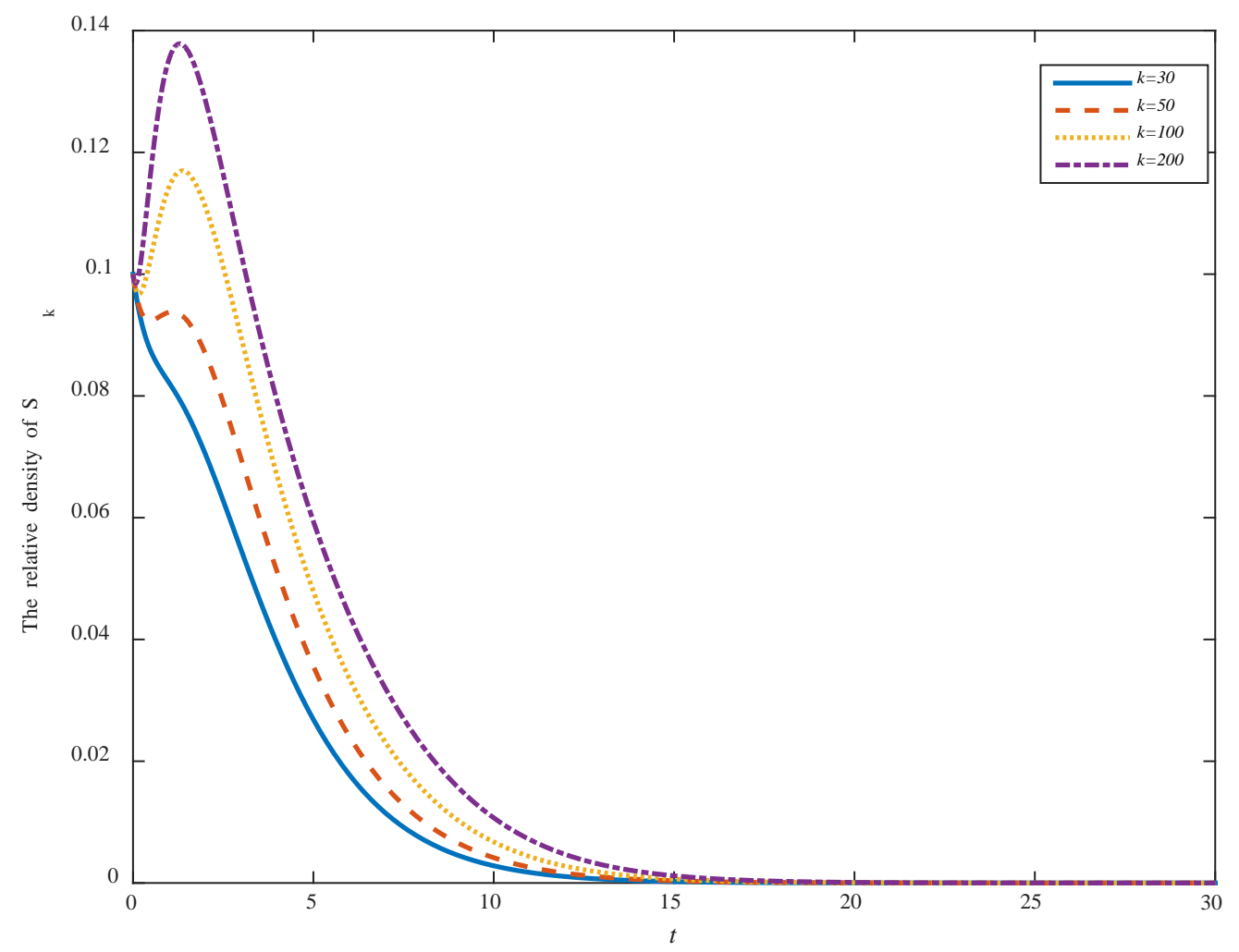

Figure 5. Dynamic behavior of light smoker with different degree when $R_{0}>1$.

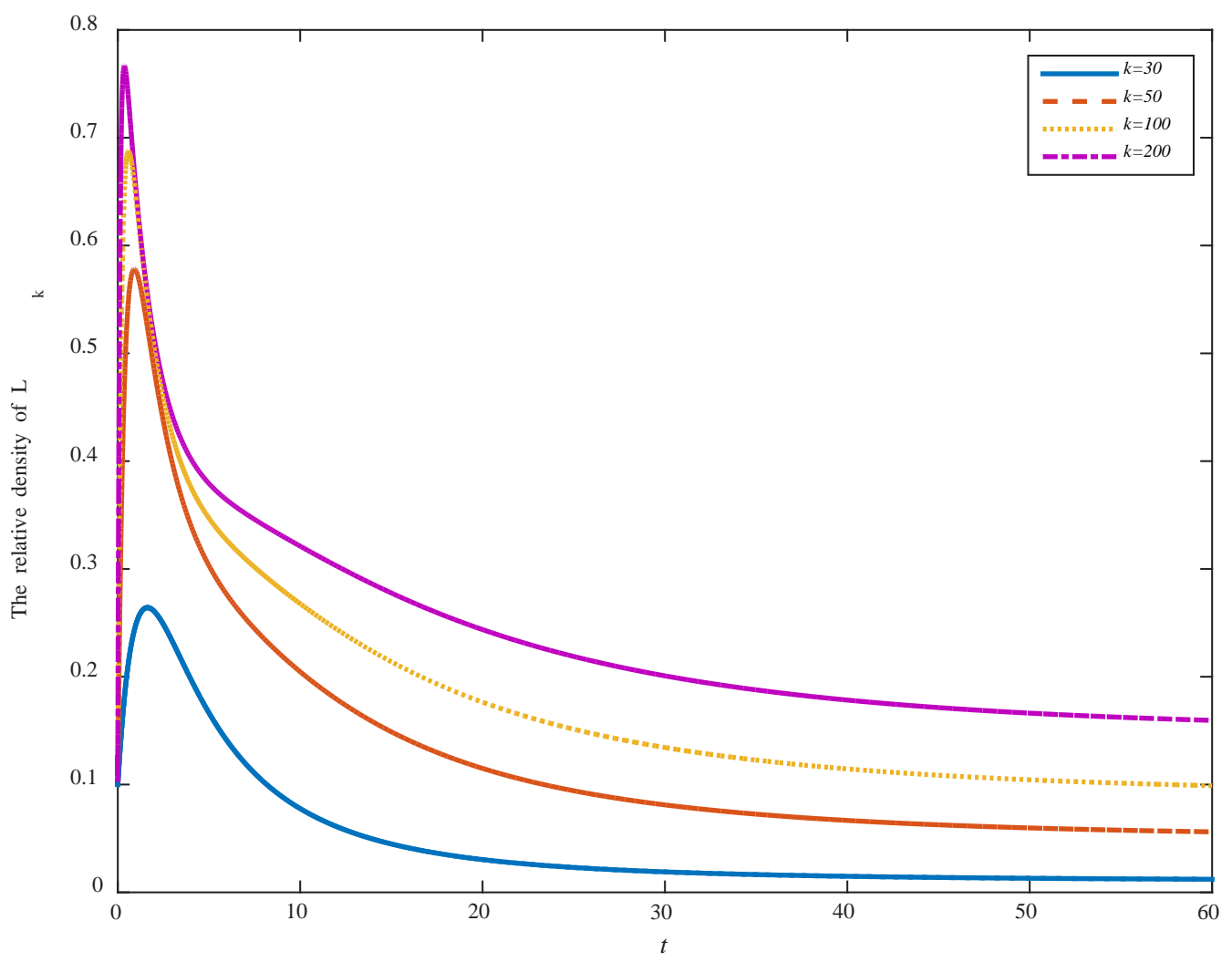

Figure 6. Dynamic behavior of light smoker with different degree when $R_{0}>1$. 


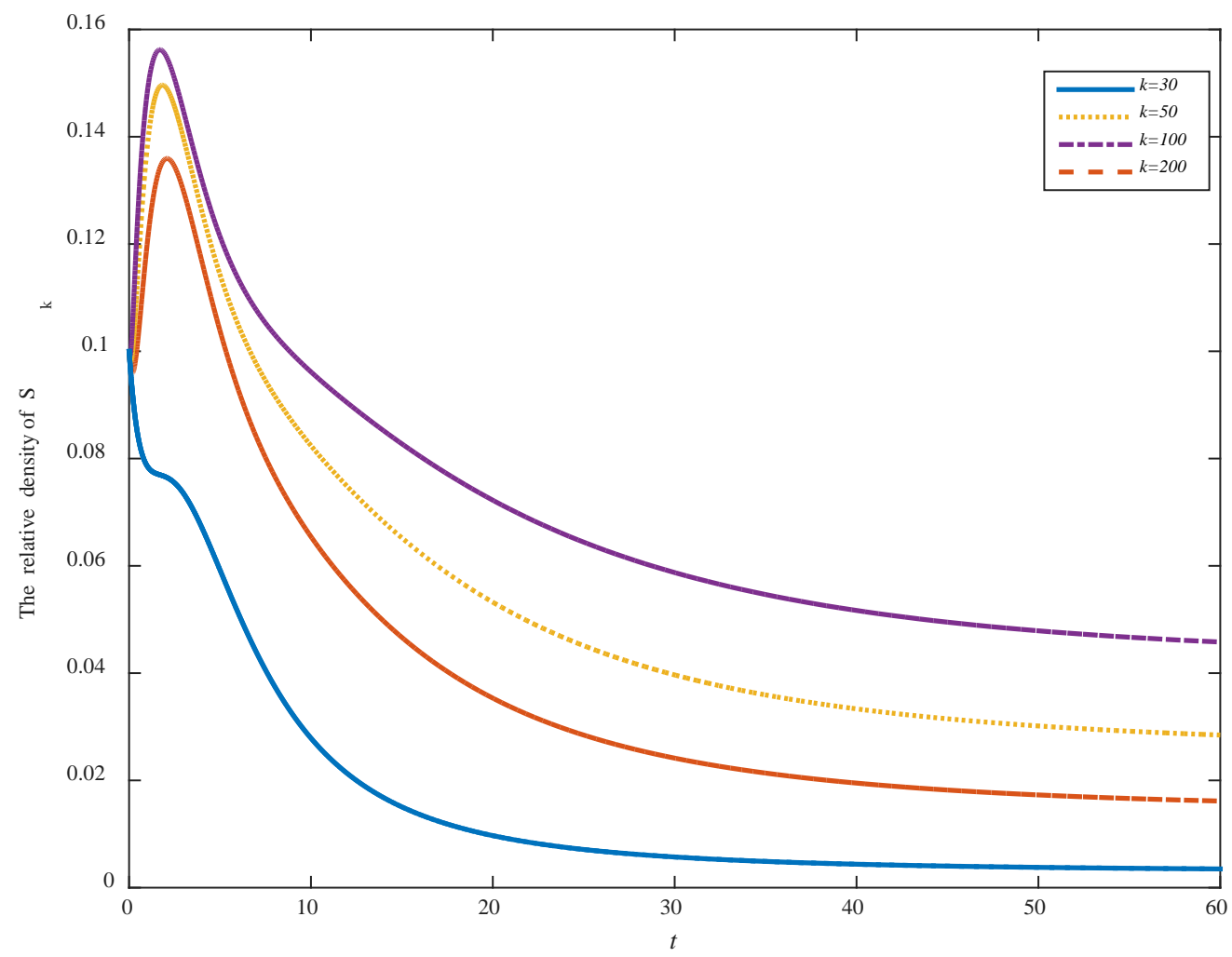

Figure 7. Dynamic behavior of light smoker with different degree when $R_{0}>1$.

We divide the smoker into two groups, light smoker and persistent smoker, considering individual's birth and death rates. Through the mathematical calculation, we obtain the basic reproduction number and equilibriums. Using the comparison theorem and the iteration principle, we analyze the stability of the smoking free equilibrium, and also give the persistence and global attractivity of the smoking. If $R_{0}<1$, the smoking-free equilibrium of the model is globally stable and smoking will disappear. If $R_{0}>1$, the smoking-present equilibrium is global attractivity and maintaining a positive constant. Furthermore, the dynamic behavior has been analyzed in our model. The study may give us valuable guiding in effectively controlling the behavior of smoking.

\section{Acknowledgements}

This work is supported by the National Natural Science Foundation of China under Grants 61672112 and Project in Hubei province department of education under Grants B2016036.

\section{References}

[1] Korobeinikov, A. (2004) Global Properties of Basic Virus Dynamics Models. Bulletin of Mathematical Biology, 66, 879-883.

https://doi.org/10.1016/j.bulm.2004.02.001

[2] Souza, M.O. and Zubelli, J.P. (2011) Global Stability for a Class of Virus Models with Cytotoxic Tlymphocyte Immune Response and Antigenic Variation. Bulletin 
of Mathematical Biology, 73, 609-625. https://doi.org/10.1007/s11538-010-9543-2

[3] Huo, H.F., Dang, S.J. and Li, Y.N. (2010) Stability of a Two-Straintuberculosis Model with General Contact Rate. Abstract and Applied Analysis, 2010, Article ID: 293747.

[4] Castillo-Chavez, C. and Song, B.J. (2004) Dynamical Models of Tuberculosis and Their Applications. Mathematical Biosciences and Engineering, 1, 361-404. https://doi.org/10.3934/mbe.2004.1.361

[5] Huo, H.F. and Feng, L.X. (2013) Global Stability for an HIV/AIDS Epidemic Model with Different Latent Stages and Treatment. Applied Mathematical Modelling, 37, 1480-1489. https://doi.org/10.1016/j.apm.2012.04.013

[6] Xu, R. (2011) Global Stability of an HIV-1 Infection Model with Saturation Infection and Intracellular Delay. Journal of Mathematical Analysis and Applications, 375, 75-81. https://doi.org/10.1016/j.jmaa.2010.08.055

[7] Pastor-Satorras, R. and Vespignani, A. (2001) Epidemic Dynamics and Endemic States in Complex Networks. Physical Review E, 63, Article ID: 066117. https://doi.org/10.1103/PhysRevE.63.066117

[8] Liu, X., Li, T., Wang, Y., et al. (2017) An SIS Epidemic Model with Infective Medium and Feedback Mechanism on Scale-Free Networks. Open Access Library Journal, 4: e3598. https://doi.org/10.4236/oalib.1103598

[9] Moreno, Y., Pastor-Satorras, R. and Vespignani, A. (2002) Epidemic Outbreaks in Complex Heterogeneous Networks. The European Physical Journal B, 26, 521-529.

[10] Li, C.H., Tsai, C.C. and Yang, S.Y. (2014) Analysis of Epidemic Spreading of an SIRS Model in Complex Heterogeneous Networks. Communications in Nonlinear Science and Numerical Simulation, 19, 1042-1054. https://doi.org/10.1016/j.cnsns.2013.08.033

[11] Chen, L. and Sun, J. (2014) Global Stability and Optimal Control of an SIRS Epidemic Model on Heterogeneous Networks. Physica A: Statistical Mechanics and Its Applications, 410, 196-204. https://doi.org/10.1016/j.physa.2014.05.034

[12] Liu, J. and Zhang, T. (2011) Epidemic Spreading of an SEIRS Model in Scale-Free Networks. Communications in Nonlinear Science and Numerical Simulation, 16, 3375-3384. https://doi.org/10.1016/j.cnsns.2010.11.019

[13] Li, T., Wang, Y. and Guan, Z.H. (2014) Spreading Dynamics of a SIQRS Epidemic Model on Scale-Free Networks. Communications in Nonlinear Science and $\mathrm{Nu}$ merical Simulation, 19, 686-692. https://doi.org/10.1016/j.cnsns.2013.07.010

[14] Huang, S., Chen, F. and Chen, L. (2017) Global Dynamics of a Network-Based SIQRS Epidemic Model with Demographics and Vaccination. Communications in Nonlinear Science and Numerical Simulation, 43, 296-310. https://doi.org/10.1016/j.cnsns.2016.07.014

[15] Castillo-Garsow, C., Jordan-Salivia, G. and Rodriguez-Herrera, A. (2000) Mathematical Models for the Dynamics of Tobacco Use, Recovery, and Replase. Tech. Rep. BU-1505-M, Cornell University, Ithaca.

[16] Zaman, G. (2011) Qualitative Behavior of Giving up Smoking Models. Bulletin of the Malaysian Mathematical Sciences Society, 34, 403-415.

[17] Zaman, G. (2011) Optimal Campaign in the Smoking Dynamics. Computation and Mathematical Methods in Medicine, 2011, Article ID: 163834.

[18] Lajmanovich, A. and Yorke, J.A. (1976) A Deterministic Model for Gonorrhea in a Nonhomogenous Population. Mathematical Biosciences, 28, 221-236.

https://doi.org/10.1016/0025-5564(76)90125-5 
[19] Chen, F. (2005) On a Nonlinear Nonautonomous Predator-Prey Model with Diffusion and Distributed Delay. Journal of Computational and Applied Mathematics, 180, 33-49. https://doi.org/10.1016/j.cam.2004.10.001 